\title{
Modelación matemática en el aula clase: una producción de modelos lineales desde el contexto del cultivo de plátano ${ }^{1}$
}

\author{
Mathematical modeling class in the classroom: a production \\ of linear models from the context of plantain
}

\section{Classe de modelagem matemática na sala de aula: a produção de modelos lineares a partir do contexto de banana}

Recibido: mayo de 2013

Aprobado: agosto de 2013
José Luis Bossio Vélez ${ }^{2}$

Sandra Milena Londoño Orrego ${ }^{3}$

Carlos Mario Jaramillo López ${ }^{4}$

\section{Resumen}

Este artículo presenta algunos resultados de investigación, que se viene desarrollando bajo el método de estudio de caso en una institución rural de la Región de Urabá, con el propósito de analizar un proceso de modelación matemática. Esto fue posible, al permitirles a los estudiantes generar modelos lineales desde una situación en el contexto del cultivo plátano. Y al final, se presentan algunos resultados, resaltando el papel del contexto cotidiano incluido en la enseñanza de las matemáticas, para mediar el uso de las letras como variables, en correspondencia entre el contexto cotidiano y las matemáticas.

Palabras clave: Aprendizaje; procesos cognitivos; modelación matemática; modelos lineales; contexto cotidiano; matemáticas y otras disciplinas; cultivo de plátano; variable.

\begin{abstract}
This article presents some results of research, which is being developed under the case study method on a rural institution Urabá region, in order to analyze a mathematical modeling process. This was possible, to allow students to generate linear models from a situation in the context of the banana crop. And finally, we present some results, highlighting the role of context in everyday inclusive teaching mathematics to mediate the use of letters as variables in the context of everyday correspondence and mathematics.
\end{abstract}

Palabras clave: slearning, cognitive processes, mathematical modeling, linear models everyday context, mathematics and other disciplines plantain; variable.

1 Artículo de Investigación

2 Institución Educativa el Dos, Turbo - Antioquia, Colombia. Contacto: Copiando2010@gmail.com

3 Universidad de Antioquia. Medellon. Colombia. Contacto: samyjdam@gmail.com

4 Instituto de Matemáticas. Universidad de Antioquia. Medellin, Colombia. Contacto: cama@matematicas.udea.edu.co Docente del 


\section{Resumo}

Este artigo apresenta alguns resultados da pesquisa, que está sendo desenvolvido sob o método de estudo de caso em uma região rural instituição Urabá, a fim de analisar um processo de modelagem matemática. Isto foi possível, para permitir que os estudantes para gerar modelos lineares a partir de uma situação no contexto da bananeira. E, finalmente, apresentamos alguns resultados, destacando o papel do contexto no cotidiano, inclusive o ensino da matemática para mediar o uso de letras como variáveis no contexto da correspondência diária e matemática.

Palabras clave: aprendizagem, processos cognitivos, modelagem matemática, o contexto de modelos lineares todos os dias, matemática e outras disciplinas banana; variável.

\section{Presentación del problema}

El lenguaje algebraico entendido como una forma particular de representación matemática, diseñado a través de símbolos para generalizar las diferentes operaciones que se desarrollan dentro de la aritmética. En este sentido, los símbolos adquieren las propiedades algebraicas de los objetos matemáticos al cual representan. Por ejemplo la "x", en el algebra elemental, según Trigueros, Ursini, \& Lozano (2000) es usada como variable y conceptualizada como incógnita o número general o en relación funcional. En esta dirección, a nuestro modo de ver, cuando a los estudiantes se les fortalece el uso de la variable como incógnita más que las otras conceptualizaciones, se estaría proyectando a los estudiantes una enseñanza de las matemáticas como un campo que se dedica a realizar operaciones con letras y números, con una exigencia de aprenderse los algoritmos para la solución de problemas.

Cabe señalar que, identificar el uso de la variable en diferentes conceptualizaciones se convierte en un obstáculo para el estudiante, porque desde la mirada de Trigueros, Ursini, \& Lozano (2000), el estudiante debe tener la habilidad de trabajar con la variable como un ente matemático integrado, cuando la usa en una situación específica, posando de uno a otros de sus aspectos (incógnita, número general, relación funcional) de manera flexible y los integra como componentes de un mismo ente matemático. Por lo anterior, podemos decir que, cuando el estudiante hace uso de la variable y al no encontrar un sentido, él le otorga una concesión errónea al manipular expresiones algebraicas (González \& Diez, 2002), asignando reglas diferentes de las operaciones estipuladas por la lógica del lenguaje algebraico. En este sentido, comprendemos la necesidad de permitirle a los estudiantes crear una correspondencia entre las situaciones del contexto cotidiano y las matemáticas a través del uso de las letras como variables, con el propósito que el contexto le posibilita la manera de reconocer el uso adecuado.

Por lo tanto, en nuestro estudio, se les ha permitido a los estudiantes desarrollar un proceso de modelación matemática en el aula de clase, a partir de las situaciones en el contexto del cultivo de plátano, con el propósito que el contexto le posibilitara mediar un uso adecuado de las letras como variable al generar modelos lineales, y a la vez, apoyar a las familias de la comunidades campesinas en las soluciones de problemas. Por tanto, la pregunta que ha orientado este estudio es ¿De qué manera un grupo de estudiantes del grado décimo, generan modelos lineales en un proceso de modelación matemática desde el contexto del cultivo de plátano?

\section{Objetivo}

Analizar un proceso de modelación matemática en estudiantes del grado décimo cuando generan modelos lineales desde el contexto del cultivo de plátano. 


\section{Objeto de estudio}

Un proceso de modelación matemática cuando se generan modelos lineales desde el contexto del cultivo de plátano.

\section{Marco de referencia conceptual}

Hoy en día, se han venido desarrollando estudios cuyo propósito es cualificar el proceso de enseñanza y aprendizaje de las matemáticas a partir de las necesidades e intereses del estudiante, en estrecha relación con su vida cotidiana, o dicho de otra manera, en los currículos, incorporarle conceptos contextualizados en la cual le permite al estudiante comprender de manera flexible el conocimiento necesario para dar solución a los problemas de situaciones cercanas a su entorno, motivando al uso pertinente de las matemáticas de manera implícita o explícita.

Una manera de contextualizar la enseñanza de las matemáticas, consiste en abordar problemas en contextos cotidianos para posibilitar los procesos de construcción de modelos matemáticos en un grupo de estudiantes. Esta construcción se corresponde con varias etapas, desde el planteamiento del problema hasta la construcción, evaluación, validación y modificación del modelo (Villa-Ochoa, J. A., 2007). De acuerdo con Villa-Ochoa, J. A., Bustamante, C. A., Berrio, M., Osorio, J. A., \& Ocampo, D. A. (2009a), quienes definen un modelo matemático como "un conjunto de símbolos y relaciones matemáticas que intentan explicar, predecir y solucionar algunos aspectos de un fenómeno o situación", es plausible considerar la idea de modelo como una construcción no inmediata que responde a un modo de ver y de representar en forma matemática un problema en particular. La situación y fenómeno que hacen parte de un mundo real desde la perspectiva de Blum, Galbraith, Henn, \& Niss, (2007) es comprendido con las relaciones que tienen las personas con la naturaleza, la sociedad o la cultura, incluyendo sobre las situaciones de la vida cotidiana, refiriéndose a los temas escolares y universitarios y las disciplinas científicas diferentes de las matemáticas. Desde estas consideraciones, cultivar plátano y exportarlo sería un trozo de la realidad, que le permitiría al estudiante la construcción de modelos lineales al intentar relacionar variables de este fenómeno y otras situaciones en contexto.

\section{Metodología}

Este trabajo de investigación está siendo abordado bajo el enfoque cualitativo de estudio de casos (Stake, 1999). Hemos considerado dicho enfoque para analizar las distintas relaciones a profundidad sobre la manera en que los estudiantes desarrollan un proceso de modelación para generar modelos lineales, a partir de una situación del contexto. De igual manera, consideramos un grupo de estudiantes que cursan el grado décimo y, trabajan el cultivo de plátano y conocen su proceso de exportación. Por lo tanto, creemos pertinente en este estudio, enfocarnos en las particularidades, de las cuales se generan un conjunto diverso de expresiones matemáticas ligado a un proceso de modelación matemática. Estas particularidades dependen de unas construcciones propias de los estudiantes, desarrollados desde las experiencias y prácticas en el contexto sociocultural.

\section{Análisis de datos}

Para este análisis, nos hemos apoyado en el software Atlas.ti, en el cual nos facilita el análisis cualitativo. Después de ir recopilando la información mediante entrevistas, observaciones y documentos escritos, para luego asumir la responsabilidad de interpretar lo que se observa, escucha o lee. Para estas interpretaciones, los datos fueron codificados, categorizadas y tematizados; con la ayuda de la propiedad del software llamada construcción de redes, esto admite crear los esquemas interpretativos permitiendo que emerjan los significados de las situaciones de mayor frecuencia. De este modo, ha facilitado la triangulación para apoyar la redacción del informe final.

\section{Conclusiones}

La solución de problemas en una situación particular, enmarcadas en el proceso de modelación matemática, les posibilitó a los estudiantes relacionar una situación en el contexto cotidiano y las matemáticas. 
Esta interconexión experimentada por los estudiantes los direccionó a la construcción de un modelo lineal, proporcionándole argumentos para tomar decisiones frente a un determinado problema. En lo anterior, está incluido el uso de las letras " $\mathrm{x}$ " $\mathrm{y}$ " $\mathrm{y}$ " como variables, al crear una correspondencia con los significados del contexto del cultivo de plátano y las matemáticas, y además, estas dos variables fueron relacionadas por el estudiante de la siguiente manera: a la variable "x" la nombró "cajas", describiendo cada caja exportada, con una constante de proporcionalidad de $\$ 16.789$ peso (precio de venta de cada caja); y a la variable "y" la nombró "precio de las cajas", describiendo el ingreso pagado por la comercializadora por las caja exportadas. Esto se puede observar de la siguiente manera:

Ezel (pseudonombre del estudiante): en este caso estamos trabajando el valor de la caja a $\$ 16.789$ peso, entonces hicimos una fórmula para la venta de $\mathrm{y}=\$ 16.789(\mathrm{x})$. Lo que tratamos es de hacer con los datos es construir una gráfica y encontrar el punto donde chocan los gastos y los ingresos, y que pasaba ahí cuando se encontraban en un punto, o sea que, de ahí para allá, obviamente, es lo que le llamamos nosotros como ganancia, si sabemos esa cantidad de gastos, obviamente, ejemplo, con 30 cajas tengo para cubrir los gastos, eso lo que necesitamos es trazar la lineal y que pasa de aquí para allá, eso es lo que pensamos hacer, cuantas cajas necesitamos para librar los gastos y que pasa de ahí para allá.

Fuente: Comentario de Ezel. Uso de las letras e idea de construcción de gráfica

Figura 1: Uso de las letras como variables: documento escrito Ezel

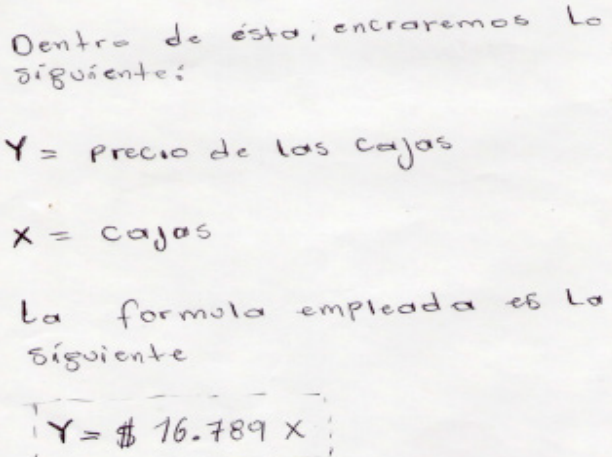

Fuente: Elaboración propia
Por lo tanto, al desarrollar un proceso de modelación matemática en el aula de clase, desde el contexto del cultivo de plátano para generar un modelo lineal, le ha permitido a los estudiantes el empoderamiento del conocimiento correspondiente, al observar y analizar los cambios en las variables inmersas en la situación de su contexto, construyendo una correspondencia entre las variables (letras), su contexto cotidiano y las matemáticas; con el propósito de deducir la ganancia de su parcela familiar. Y, adoptando una posición crítica frente a situaciones de su contexto sociocultural.

\section{Referencias}

Blum, W., Galbraith, P. L., Henn, H. W., \& Niss, M. (Eds.) (2007). Modelling and Applications in Mathematics Education: The 14th ICMI Study. Springer.

González, F. E., \& Diez, M. M. (2002). Dificultades en la adquisición del significado en el uso de las letras en Álgebra. Propuesta para la interacción didáctica, 13, 281-302.

Stake. R. E. (1999). Investigación con estudio de casos. Madrid: Morata.

Trigueros, M., Ursini, S. \& Lozano, D. (2000). La conceptualización de la variable en la enseñanza media. Educación Matemática, 12 (2), 27-48.

Villa-Ochoa, J. A. (2007). La modelación como proceso en el aula de matemáticas. Un marco de referencia y un ejemplo. Tecno Lógicas, (19) pp.51-81.

Villa-Ochoa, J. A., Bustamante, C. A., Berrio, M., Osorio, J. A., \& Ocampo, D. A. (2009a). Sentido de realidadymodelación matemática. El caso de Alberto. ALEXANDRIA. Revista de Educação em Ciência e Tecnologia, 2 (2), 159-180. 